\title{
Der Maskenverweigerer
}

blog.kulturwissenschaften.de/der-maskenverweigerer/

\section{$\underline{\text { Marion Müller }}$}

\section{Die Suche nach einem Schurken in der Pandemie}

\section{Urbane Pandemie-Mythen}

Immer wieder werden sie erzählt oder man liest sie in der Zeitung: kurze Geschichten von so genannten „Maskenverweigerern“. ${ }^{1}$ Gemeint sind Menschen, die entweder gar keinen Mund-Nasen-Schutz tragen oder diesen nur halbherzig anlegen: mit heraushängender Nase, Maske unterm Kinn oder am Ohr baumelnd. ${ }^{2}$ Oft ist unklar, ob es sich um grundsätzliche Verweigerung handelt oder eher um eine (mehr oder weniger charmant inszenierte) kalkulierte Form der Nachlässigkeit gegenüber diesem neuen, unbeliebten Alltagsaccessoire. Entscheidend für die Identifikation der Intention hinter der Maskenlosigkeit ist die Reaktion der betroffenen Person auf Ermahnungen. Hier offenbaren sich die wirklich Widerständigen, um die es in diesen Gruselgeschichten eigentlich geht, ziehen doch diejenigen, die die Maske einfach nur vergessen haben, sie spätestens nach einer expliziten Aufforderung ohne weitere Diskussionen auf.

Hintergrund dieser neuen urban myths ist die Einführung einer Maskenpflicht in vielen Ländern der Welt im Zuge der Corona-Pandemie: Sie bezieht sich meistens auf einen nicht-medizinischen Mund-Nasen-Schutz und gilt je nach Land überall in der Öffentlichkeit oder nur für bestimmte Situationen, wie beim Betreten von Läden oder im öffentlichen Nahverkehr. ${ }^{3}$

Gemeinsam ist diesen Geschichten über Maskenverweigerer vor allem die rechtschaffene Empörung, die sie bei den Nicht-Verweigerern auslöst. Das gilt sowohl für die sich in der beschriebenen Situation befindlichen Personen als auch für die Leser*innen und Zuhörer*innen. Die Geschichten bestehen letztlich aus relativ wenig Handlung und fast ausschließlich aus der Feststellung des Verstoßes und der Reaktion der anderen Anwesenden. So z. B. die Berichte über die Mitglieder der AfDFraktion im hessischen Landtag, die sich der „dringenden Empfehlung“ zum Maskengebrauch verweigern. ${ }^{4}$ Demnach seien alle Appelle an „Kollegialität“ und „Verantwortungsgefühl“ durch den Parlamentspräsidenten vergeblich geblieben. Lediglich ein Abgeordneter habe auf die direkte Bitte seines unmittelbaren Sitznachbarn reagiert, „ihm Respekt [zu] zollen und heute eine Maske [zu] tragen“. ${ }^{5}$

Die entrüsteten Reaktionen in den sozialen Medien zeigen, dass sich offenbar nicht nur die persönlich Anwesenden vom Fehlen einer Maske bedroht fühlen, sondern auch die Personen, die sich nur Videos des Geschehens anschauen. Das belegen die 
zahlreichen Posts von Fotos und Videos von Menschen mit fehlerhaften oder gar keinen Masken, über die sich kollektiv empört wird. ${ }^{6}$ Die Wut und Verärgerung ist enorm und wird ungehemmt geäußert: So z. B. auf Twitter unter dem Hashtag „\#Maskenverweigerer“, wo selbige als „potentielle Mörder"7 und „Kriminelle“8 beschimpft werden. Selbst in den Kommentarspalten seriöser Medien finden sich ähnlich drastische Stellungnahmen, wie z. B. von Samira El Ouassil im „Spiegel“: „Es ist grob unhöflich, andere Menschen umbringen zu wollen. Es ist praktisch allen respektlos gegenüber (...). Jedem atmenden Menschen gegenüber ist es respektlos. Warum also wollen Sie ein aerosoler Rüpel bleiben?"99

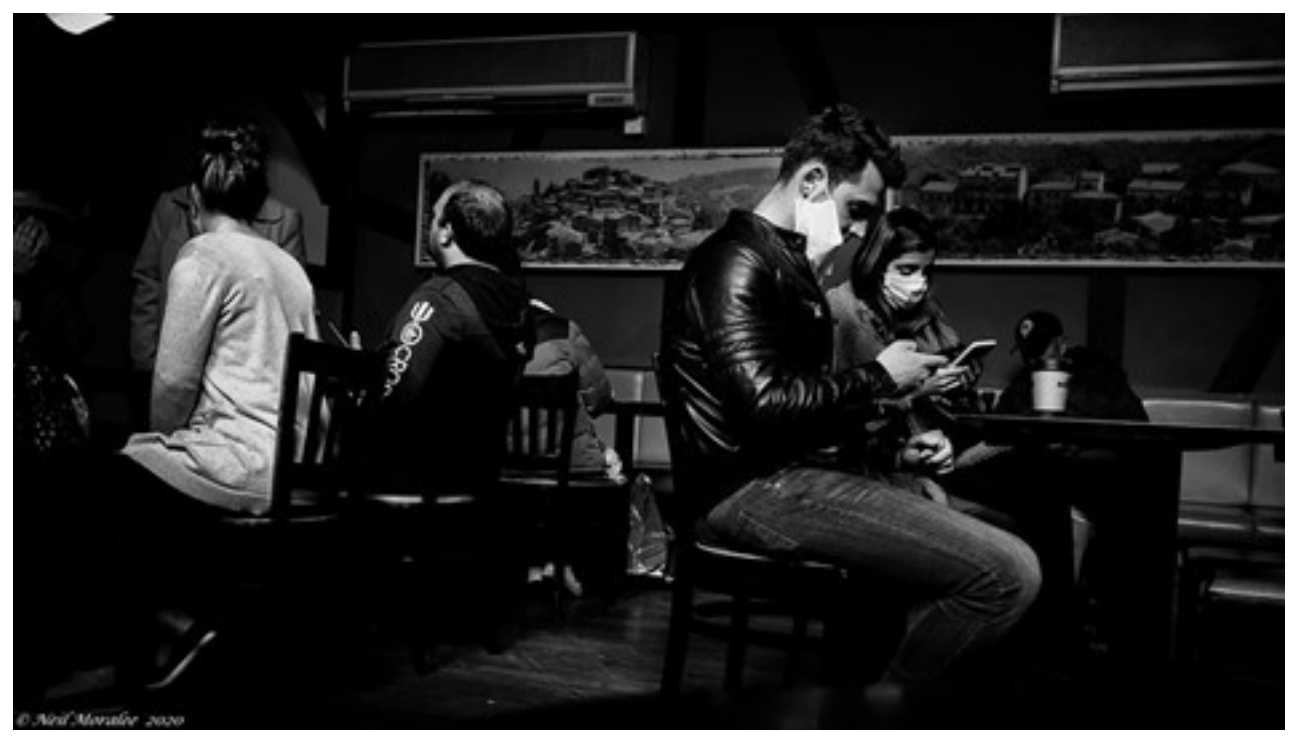

Abb. 1: „Masks and Cold Coffee” des Fotografen Neil Moralee, https://www.flickr.com/photos/neilmoralee/50510948556/, is licensed under CC BY-NC-ND 2.0

\section{Maskenverweigerung: eine kriminelle Unterlassung?}

Maskenverweigerer werden fast durchgängig als dümmlich-egoistische und gefährliche Abweichler dargestellt, denen die eigene Bequemlichkeit wichtiger ist als das Wohlergehen anderer. Das Verweigern der Maske wird den betreffenden Personen also als absichtsvolles Handeln zugerechnet, als ein Unterlassen aus Faulheit oder Gleichgültigkeit sowie ein Billigend-in-Kauf-Nehmen möglicher negativer Folgen für sich und vor allem für andere. Das Maske-Tragen erscheint dagegen nicht nur als Pflicht, sondern als „Dienst an der Allgemeinheit" ${ }^{\text {“10 }}$, als eine aktive Form des Schutzes anderer. So berichtet der Spiegel über ein Erlebnis in der Bahn und zitiert den Schaffner, der nach Ansicht des Autors „ein wahres Wort“ spricht, nachdem er sieht, dass die Leute erst bei der Fahrkartenkontrolle ihre Masken richtig anziehen: „Wegen mir müssen Sie Ihre Masken nicht aufsetzen. Es geht hier nicht um irgendeine bescheuerte Schikane der Deutschen Bahn, sondern um Ethik. Sie schützen Ihre Mitmenschen und sich selbst. Das nicht zu tun, ist asozial.“11 
Neben den recht harmlosen Geschichten der Verweigerung gibt es auch brutalere, in denen sich die Figur des Maskenverweigerers als renitenter Querulant mit einer Neigung zur Gewalttätigkeit entpuppt. So z. B. in Hagen, wo ein Mann eine Frau in einer Bäckerei beleidigte und bespuckte, „weil sie ihn auf die Maskenpflicht hingewiesen" hatte. ${ }^{12}$ Oder der "gemeinschaftliche Angriff" von fünf Personen ohne Masken in einer S-Bahn zwischen Wattenscheid und Essen auf einen 52-jährigen Mitreisenden, nachdem dieser „die Gruppe auf die Maskenpflicht in Zügen aufmerksam gemacht" hatte. ${ }^{13}$ Laut Polizeibericht schlugen sie daraufhin auf ihn ein und würgten ihn.

An dieser Stelle sei kurz erwähnt, dass die Sozialfigur des Maskenverweigerers keineswegs exklusiv männlich ist, selbst wenn sich die meisten Geschichten auf Männer beziehen. Hin und wieder - man hat den Eindruck mit zunehmender Häufigkeit - finden sich auch Beispiele maskenverweigernder (gewalttätiger) Frauen: z. B. Jayla und Jessica Hill, die in einem Geschäft in Chicago einen Wachmann mit 27 Messerstichen schwer verletzten, als er sie aufforderte, eine Maske anzulegen. ${ }^{14}$

Wenig überraschend vermischt sich die Empörung über Maskenverweigerer manchmal mit der Entrüstung über andere Personengruppen, über die sich auch außerhalb der Corona-Pandemie bereits aufgeregt wird. Man könnte sagen: Es handelt sich um die „üblichen Verdächtigen“. So liest man z. B. in einer Polizeimeldung aus Konstanz über einen Mann, der sich im Bus weigerte, eine Maske zu tragen, den Fahrer beschimpfte und sich anschließend der Verhaftung widersetzte: „Der Tatverdächtige stammt aus Westafrika. Er dürfte noch nicht erkannt haben, dass Busfahrer und ähnliche Leistungsträger in Mitteleuropa im Gegensatz zu seiner Heimat mehr als systemrelevant und weniger als minderwertig angesehen werden."15

Ähnliche Ressentiments ließ die kollektive Empörung über eine türkische Großhochzeit Anfang September in Hamm anklingen, bei der niemand eine Maske getragen hatte und sich letztlich mehr als 200 Personen mit Covid-19 infizierten. „Es kann nicht sein, dass eine einzige Familie mit ihrer Familienfeier eine ganze Stadt durcheinanderbringt oder im schlimmsten Fall sogar noch einen Lockdown auslöst" ${ }^{16}$ hatte sich Hamms Oberbürgermeister echauffiert. In der Folge entstand eine Art Generalverdacht gegenüber „arabischen Hochzeiten in CoronaZeiten "17 inklusive der bekannten Stereotypen, mit denen muslimische Mitbürger*innen belegt werden. Bereits vorhandene soziale Unterscheidungen werden hier von neuen „pandemischen Humandifferenzierungen“ überlagert, denen zufolge Mitmenschen entlang der Kategorien „Gefährder vs. Gefährdete“ sowie „Verantwortungsvolle vs. Leichtsinnige“ wahrgenommen werden. ${ }^{18}$ 


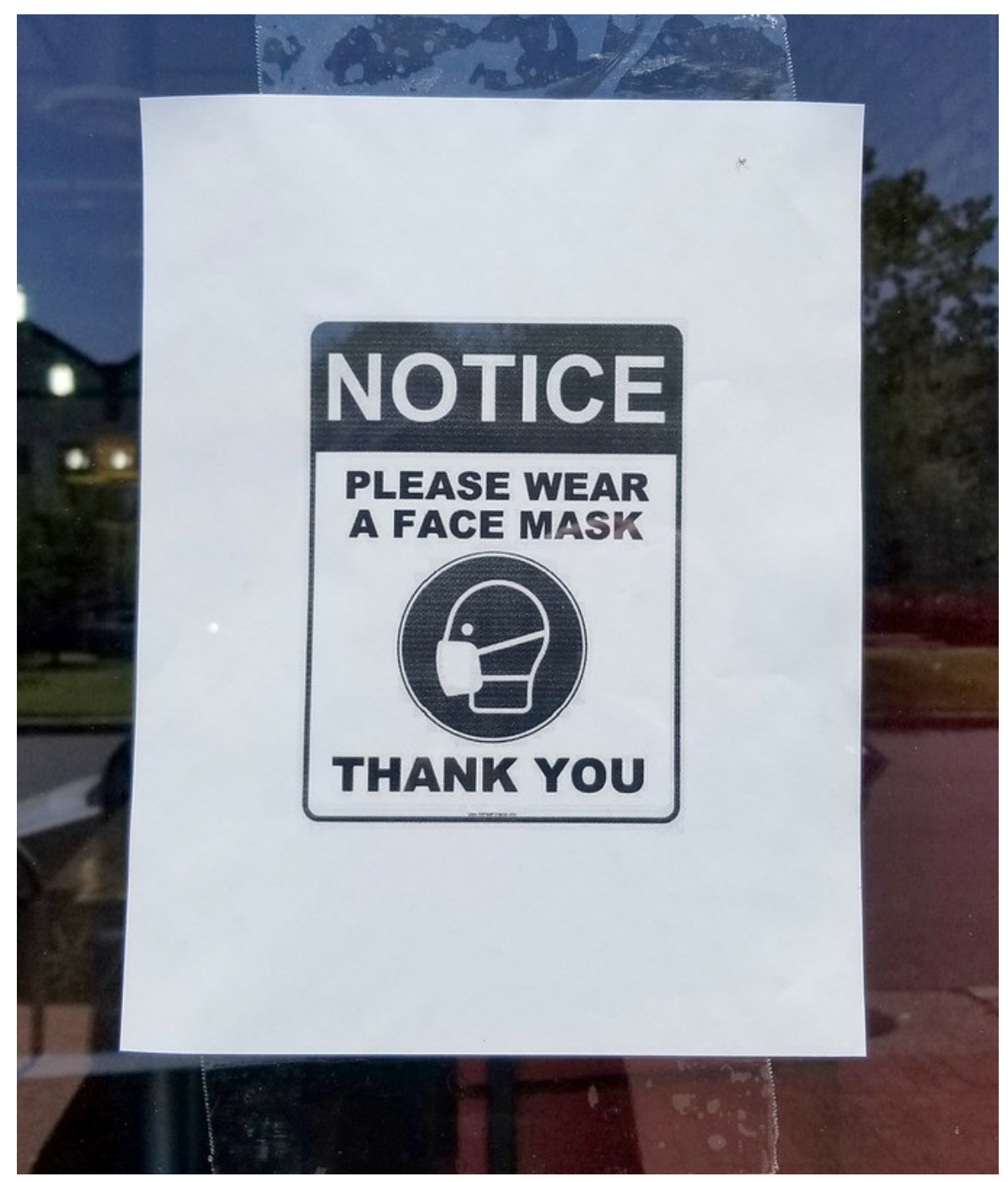

Abb. 2: "Face Mask Sign" by Mike Licht, NotionsCapital.com is licensed under $\underline{\text { CC BY } 2.0}$

\section{Vom Umgang mit den Schurken}

Seit Ausbruch der Corona-Pandemie scheinen Menschen sich und vor allem ihre Körper wechselseitig primär als potentielle Bedrohung zu betrachten.

Grunderfahrungen von Verwundbarkeit und Gefahr waren zwar immer mit unmittelbaren Begegnungen verbunden, das Risiko dieser Verletzbarkeit schien aber (wenn auch je nach sozialer Zughörigkeit unterschiedlich) in einem Rechtsstaat wie dem unseren mehr oder weniger gut kalkulierbar zu sein. Nun sorgt die staatlich verordnete Maskenpflicht jedoch für eine Art „diktiertes

Misstrauen"19 untereinander, das sich u. a. in zahlreichen Ratgeber-Artikeln zum Umgang mit Maskenverweigerern im Alltag widerspiegelt. Hier erklären z. B. Psycholog*innen oder Konflikt-Coaches, wie man am besten bei solchen Begegnungen reagieren sollte. Allerdings erscheint es kaum möglich das Fehlen der Maske zu thematisieren, ohne dabei vorwurfsvoll oder belehrend zu klingen. Und da sich niemand gerne von anderen Menschen (noch dazu Fremden und in der Öffentlichkeit) maßregeln lässt, enden solche Aufforderungen nicht selten in Konflikten: Letztlich berichten viele Maskenverweigerer-Geschichten genau davon. Besagte Konfliktexpert*innen empfehlen denn auch, auf jede Art von 
Vorwurfskommunikation zu verzichten und stattdessen „Ich-Botschaften“ $\mathrm{zu}$ senden..$^{20}$ Oder aber man solle Sätze sagen wie: „Ich sehe, Sie haben keine Maske auf. Mir geht es schlecht damit. “21 Der gleiche Mediator empfiehlt außerdem, sich nicht auf inhaltliche Diskussionen einzulassen und stattdessen lieber zu sagen: „Es ist möglich, dass Sie recht haben. Vielleicht habe auch ich recht. Ich weiß es nicht.“ Alternativ solle man die Debatte lieber auf sich und eine emotionale Ebene zurückbringen und Sätze sagen wie: „Wissen Sie, ich habe Angst, wenn ich auf engem Raum mit Menschen zusammen bin, die keine Maske tragen.“22

\section{Moralisierungen und Schuldzuweisungen}

In der Sozialfigur des Maskenverweigerers verdichtet sich die große Unsicherheit, mit der wir derzeit in unserem Alltag zurechtkommen müssen. Wir leben unter der Bedingung des Nicht-Wissens bzw. der ständigen Veränderung von Wissensbeständen über das Virus, dessen Übertragungswege und mögliche Schutzmaßnahmen: Während man zunächst davon ausging, dass es nur über Schmierinfektionen übertragen werde, vermuten viele Wissenschaftler*innen nun, dass die Ansteckung ausschließlich über Aerosole erfolge. Entsprechend wurde und wird das Tragen von Maskenmal für nutzlos und mal für wichtig gehalten. Was viele Menschen außerdem verunsichert ist, dass selbst die Virolog*innen sich darüber nicht einig zu sein scheinen. Die Pandemie hat möglicherweise das Bewusstsein dafür geschärft, dass auch die Naturwissenschaften, deren Ergebnisse und Empfehlungen alltagsweltlich häufig als eindeutig und unzweifelhaft wahrgenommen werden, widersprüchliche Ergebnisse produzieren. Eng damit verbunden ist außerdem eine umfassende Neuinterpretation der persönlichen Nah- und Distanzräume sowie der damit verbundenen Interaktionsordnung: So darf man unter Pandemiebedingungen auch relativ vertrauten Personen nicht mehr so nah kommen und körperliche Distanzierung ist plötzlich die neue Form der Höflichkeit. Diese neue Norm der Rücksichtnahme im direkten Kontakt bringt der Virologe Christian Drosten mit seinem „pandemischen Imperativ“ folgendermaßen auf den Punkt: „Handle in einer Pandemie stets so, als seist du positiv getestet und dein Gegenüber gehörte einer Risikogruppe an". ${ }^{23}$

Die Sozialfigur des Maskenverweigerers nimmt in dieser besonderen Gemengelage aus Angst, Unsicherheit und Nichtwissen in der Corona-Pandemie eine Schlüsselstellung ein: So steht im Zentrum all dieser Geschichten die klare Verurteilung von Maskenverweigerern, die sozusagen als zentrale Bösewichte der Pandemie identifiziert werden. Es wird nicht nur ihr Verhalten negativ bewertet, sondern die Missachtung richtet sich auf die ganze Person des Maskenverweigerers, die als rücksichtslos, egoistisch und asozial verurteilt wird. Die Moralisierung ist zentral für diese Geschichten: ${ }^{24}$ Sie adressieren das derzeit gesamtgesellschaftlich vorherrschende Gefühl der Unsicherheit und Angst, erleichtern aber auch deren Bewältigung, weil man das Geschehen nicht mehr nur ohnmächtig beobachten, sondern bestimmten Personen Schuld zurechnen kann. Das Virus selbst wird zwar auch gelegentlich als Täter inszeniert (z. B. durch die Meldungen täglicher 
Opferzahlen), entzieht sich aber jeder Form von Schuldzuweisungen sowie Bestrafungen. Im Gegensatz dazu kann man sich jedoch über Maskenverweigerer kollektiv empören, ihnen die Verantwortung für die Weiterverbreitung des Virus zuschreiben und sie bestrafen.

Analog ließe sich überlegen, ob die Gegenwehr der Maskenverweigerer nicht auf ein ähnliches Reaktionsmuster hinweist: Sie empören sich über die ihrer Ansicht nach unangemessene Maßnahme der Maskenpflicht, die Einschränkung ihrer Freiheiten und schreiben die Schuld dafür Politiker*innen, Mediziner*innen oder auch Unternehmer*innen wie Bill Gates zu, die sie dann verurteilen können (z. B. auf Plakaten, die Maskenverweigerer auf sog. Anti-Corona-Demos zeigen, auf denen Angela Merkel u. a. in gestreiften Häftlingsuniformen als Kriminelle dargestellt $\operatorname{sind}^{25}$ ). Offensichtlich sind dies für Maskenverweigerer die eigentlichen Bösewichte in der Pandemie. Letztlich ist die ganze Aufregung auf beiden Seiten vielleicht ein bisschen wie das Pfeifen im Walde, das uns vor allem von unseren Ängsten ablenken soll.

\section{References}

1. Diese Geschichten gibt es, ebenso wie den Begriff, in vielen anderen Ländern und Sprachen (z. B. „mask denier“ in den USA, „les anti-masques“ oder „réfractaires“ in Frankreich und „munskydd vägrare“ in Schweden, obwohl es da gar keine Maskenpflicht gibt!). Insofern scheint der Maskenverweigerer eine Sozialfigur mit globaler Reichweite zu sein.

2. Benachbarte und sich gelegentlich vermutlich auch überlagernde Sozialfiguren der Pandemie sind „Corona-Leugner“ und „Covidioten“. Noch relativ neu im Diskurs (und vermutlich eine Art Steigerungsform des Maskenverweigerers) ist der "Quarantäne-Verweigerer“.

3. Mit der Frage der tatsächlichen Effektivität der Alltagsmasken gegen eine Ansteckung mit Covid-19 beschäftigt sich dieser Beitrag nicht. Eine genauere Chronologie der wechselnden öffentlichen Empfehlungen u. a. auch zum Maske-Tragen findet man hier:

https://www.bundesgesundheitsministerium.de/coronavirus/chronikcoronavirus.html.

4. https://www.hessenschau.de/politik/landtag/masken-zoff-im-landtag-weildie-afd-oben-ohne-antritt,landtag-maskenstreit-100.html

5. Ebd.

6. Z. B. über die funktionslose Netzmaske der Sängerin Lana del Ray (https://www.t-online.de/unterhaltung/stars/id 88697196/lana-del-reysaengerin-loest-mit-netz-maske-empoerung-bei-instagram-aus.html) und über die vom WDR live übertragene Party der SPD in Köln anlässlich der Oberbürgermeisterwahl (https://www.welt.de/politik/deutschland/article216730360/Empoerungueber-SPD-Wahlparty-ohne-Masken-in-Koeln.html). 
7. https://twitter.com/gewue1/status/1293839962312507393

8. https://twitter.com/Unvernunft2/status/1320358313050148865

9. https://www.spiegel.de/kultur/corona-pandemie-brief-an-dieschutzmaskenverweigerer-a-5069f101-74aa-4dae-89a6-9aeage5331da

10. https://www.spiegel.de/wissenschaft/medizin/coronavirus-undmaskenpflicht-debatte-die-dinger-nerven-na-und-a-af3f749c-17b9-4094-9oc7975198707475

11. Ebd.

12. https://www.ruhr24.de/nrw/coronavirus-nrw-maskenpflicht-polizeimundschutz-zug-bahnhof-gewalt-angriff-nrw-dortmund-essen-13861288.html

13. https://www.presseportal.de/blaulicht/pm/70116/4681243

14. https://www.the-sun.com/news/1703955/sisters-jayla-jessica-hill-stab-guardbladed-comb/

15. https://www.swr.de/swraktuell/baden-wuerttemberg/kritik-polizeimeldungmaskenverweigerer-braeunlingen-100.html

16. https://www1.wdr.de/nachrichten/themen/coronavirus/tuerkischehochzeiten-kritik-tradition-100.html

17. https://www.welt.de/politik/ausland/article216667788/Arabische-Hochzeitenin-Corona-Zeiten-Feiern-bis-die-Polizei-kommt.html

18. Hirschauer, Stefan 2020: „Pandemische Humandifferenzierung“. In: Volkmer, Michael/Werner, Karin (Hg.): Die Corona-Gesellschaft. Bielefeld: Transcript, S. 217-225. https://doi.org/10.14361/9783839454329-022

19. Allert, Tilman 2020: Das Miteinander in der Krise. Erstarrte Begegnungen mit maskiertem Gesicht. In: Frankfurter Allgemeine Zeitung vom 25.10.2020

20. https://www.zdf.de/nachrichten/panorama/coronavirus-maske-verweigererreaktion-100.html

21. Ebd.

22. Ebd.

23. https://www.deutschlandfunkkultur.de/christian-drostens-schillerredebrauchen-wir-einen.1013.de.html?dram:article id=487183

24. Allgemein zur Bedeutung von Moralisierungen in der Pandemie vgl. Dieterich, Manuel 2020: Corona-Krise und Moralisierungen. In: Soziologie Magazin \#1 2020 (13.05.2020). URL: https://soziologieblog.hypotheses.org/13561

25. https://www.welt.de/politik/deutschland/article215026642/CoronaAerztepraesident-ruegt-Entgleisung-von-Medizinern-bei-Protest.html

SUGGESTED CITATION: Müller, Marion: Der Maskenverweigerer. Die Suche nach einem Schurken in der Pandemie, in: KWI-BLOG, [https://blog.kulturwissenschaften.de/der-maskenverweigerer/], 07.12.2020

DOI: https://doi.org/10.37189/kwi-blog/20201207-0830 


\section{DuEPublico}

Dieser Text wird über DuEPublico, dem Dokumenten- und Publikationsserver der Universität Duisburg-Essen, zur Verfügung gestellt. Die hier veröffentlichte Version der EPublikation kann von einer eventuell ebenfalls veröffentlichten Verlagsversion abweichen.

DOI: $\quad 10.37189 / \mathrm{kwi}-\mathrm{blog} / 20201207-0830$

URN: urn:nbn:de:hbz:464-20201207-101040-7 